Bull. Austral. Math. Soc.

05c25, 05-04

Vol. 55 (1997) [385-393]

\title{
GROUPS WITH A CAYLEY GRAPH ISOMORPHIC TO A HYPERCUBE
}

\author{
JOHN D. DIXON
}

\begin{abstract}
A process is described for enumerating the Cayley graphs isomorphic to a binary $d$-cube for small values of $d$. There are 4 Cayley graphs isomorphic to the 3-cube, 14 isomorphic to the 4-cube, 45 isomorphic to the 5-cube and 238 isomorphic to the 6-cube. A similar method may be used for any graph with a prime power number of vertices.
\end{abstract}

\section{INTRODUCTION}

Let $G$ be a group and $T$ be a set of generators of $G$ with $T=T^{-1}$ and $1 \notin T$. Then the Cayley graph Cayley $(G, T)$ is the graph with vertex set $G$ and edges $(x, x t)$ ( $x \in G, t \in T$ ). The graph is connected because $T$ generates $G$, it has no loops because $1 \notin T$, and it is undirected since whenever $(x, x t)$ is an edge so is $\left(x t,(x t) t^{-1}\right)=(x t, x)$. There is an extensive literature on Cayley graphs, partly because their simple representation and internal symmetry make them useful in applications (see, for example, the recent papers $[\mathbf{7}, \mathbf{8}, \mathbf{1 0}]$ and $[\mathbf{1 2}])$.

Two natural questions arise: (1) what graphs can be represented by Cayley graphs; and (2) what different representations does a given graph have? The first of these question seems quite difficult, although some progress has been made; see, for example, [9] and the references there. With respect to the second question, most effort seems to have gone into attempts to characterise "Cayley invariant groups" (CI-groups) which were introduced in [1] (the definition is given below); see $[4,5,6]$ and references there.

In the present paper we consider question (2) for $d$-cubes which are of particular interest for applications. We shall show how to compute a complete set of representations of a $d$-cube as a Cayley graph for small values of $d$. It turns out that there are many representations even when $d \leqslant 6$. Our methods apply equally well to any graph with a prime power number of vertices.

One reason that Cayley graphs are interesting is because of their in-built symmetry. If $A$ denotes the automorphism group of Cayley $(G, T)$ (so $A$ is the group of all

Received 3rd June, 1996

This research was supported in part by grant NSERC A7171. The author wishes to acknowledge the hospitality of the Department of Mathematics of the University of British Columbia where this work was done.

Copyright Clearance Centre, Inc. Serial-fee code: 0004-9729/97 \$A2.00+0.00. 
permutations of the vertex set $G$ which preserve adjacency), then $A$ contains a copy $G_{0}$ of $G$ as a regular subgroup acting on the left, namely:

$$
G_{0}:=\left\{x \mapsto a^{-1} x \mid a \in G\right\} \cong G .
$$

Our constructions below are based on the following converse.

(1.1) Let $\mathcal{G}$ be a connected graph (without loops) whose automorphism group $A$ contains a regular subgroup $G$ (in its action on the vertex set), and let $\alpha$ be a vertex of $\mathcal{G}$. Then $T:=\left\{t \in G \mid \alpha^{t}\right.$ is adjacent to $\alpha$ in $\left.\mathcal{G}\right\}$ satisfies $1 \notin T$ and $T=T^{-1}$, and $\mathcal{G}$ is isomorphic to Cayley $(G, T)$.

Proof: See [2, Lemma 16.3].

When should two Cayley graphs be considered "the same"? The following is a natural equivalence. Let $\left(G_{i}, T_{i}\right)(i=1,2)$ be two pairs where $T_{i}$ is a generating set for the group $G_{i}$ such that $1 \notin T_{i}$ and $T_{i}=T_{i}^{-1}$. We say that these pairs are equivalent if there exists a group isomorphism $\psi$ from $G_{1}$ onto $G_{2}$ which maps $T_{1}$ onto $T_{2}$. Evidently, if $\left(G_{1}, T_{1}\right)$ is equivalent to $\left(G_{2}, T_{2}\right)$ under $\psi$, then $\psi$ induces an isomorphism between Cayley $\left(G_{1}, T_{1}\right)$ and Cayley $\left(G_{2}, T_{2}\right)$. In general, pairs which are inequivalent may still have isomorphic Cayley graphs; Babai [1] defines $G$ to be a CI-group if inequivalent pairs $\left(G, T_{1}\right)$ and $\left(G, T_{2}\right)$ always have nonisomorphic Cayley graphs. The next result shows how we can determine equivalence in the situation of (1.1).

(1.2) Suppose that the automorphism group $A$ of a connected graph $\mathcal{G}$ contains two regular subgroups $G_{1}$ and $G_{2}$. Let $\alpha_{1}$ and $\alpha_{2}$ be any two vertices of $\mathcal{G}$ and define $T_{i}:=\left\{t \in G_{i} \mid \alpha_{i}^{t}\right.$ is adjacent to $\left.\alpha_{i}\right\}$ for $i=1,2$. Then $\left(G_{1}, T_{1}\right)$ is equivalent to $\left(G_{2}, T_{2}\right)$ if and only if $G_{1}$ is conjugate to $G_{2}$ in $A$. In particular, the equivalence type of $\left(G_{1}, T_{1}\right)$ does not depend on the choice of $\alpha_{1}$.

Proof: See [1].

Finally, the following criterion for equivalence is useful for computations. Its simple proof is omitted.

(1.3) Suppose that $\left(G_{i}, T_{i}\right)(i=1,2)$ are two pairs where $T_{i}$ is a generating set for the group $G_{i}$, and that $G_{1}$ is a finite group of order $g$. Then there is an isomorphism $\psi$ of $G_{1}$ onto $G_{2}$ such that $\psi\left(T_{1}\right)=T_{2}$ if and only there is a bijection $\lambda: T_{1} \rightarrow T_{2}$ such that $\left\{(t, \lambda(t)) \mid t \in T_{1}\right\}$ generates a subgroup of order $g$ in $G_{1} \times G_{2}$ (and in the latter case $\lambda$ extends to an isomorphism $\psi$ of $G_{1}$ onto $G_{2}$ ).

REMARK. In a computation to decide whether two pairs $\left(G_{1}, T_{1}\right)$ and $\left(G_{2}, T_{2}\right)$ are equivalent, we work with partial (injective) functions $\lambda: T_{1} \rightarrow T_{2}$. The criteria in (1.3) is used to check whether $\lambda$ can be extended to an isomorphism on the subgroup generated by its domain. If it is, we extend $\lambda$ so that its domain contains one further element of $T_{1}$; otherwise we backtrack by deleting an element from the domain. 
EXAMPLE 1. There are exactly four inequivalent pairs $(G, T)$ for which Cayley $(G, T)$ is isomorphic to the 3-cube. (This is not obvious but see Example 2 below.) These are:

(i) $G$ is elementary Abelian of order $2^{3}$ with $T=\{a, b, c\}: a^{2}=b^{2}=c^{2}=1$, $a b=b a, a c=c a$ and $b c=c b$ (the "standard example");

(ii) $G$ is a direct product of cyclic subgroups of order 2 and 4 with $T=$ $\left\{a, b, b^{-1}\right\}: a^{2}=b^{4}=1$ and $a b=b a$;

(iii) $G$ is dihedral of order 8 with $T=\{a, b, c\}: a^{2}=b^{2}=c^{2}=1, a b=b a$, $a c=c a$ and $b c=c b a$

(iv) $G$ is dihedral of order 8 with $T=\left\{a, b, b^{-1}\right\}: a^{2}=b^{4}=1$ and $a b=b^{3} a$. The pairs $(G, T)$ in (iii) and (iv) are obviously inequivalent even though the underlying group is the same.

\section{AUTOMORPHISMS OF THE $d$-CUBE}

We shall define the (binary) $d$-cube $(d \geqslant 2)$ as the graph whose vertex set is the vector space $V:=\left(\mathbb{F}_{2}\right)^{d}$ of $d$-tuples over the field of two elements with two vertices adjacent if and only if they differ in exactly one entry. The automorphism group $A$ of the $d$-cube clearly contains the elementary Abelian 2-group $B$ of order $2^{d}$ consisting of all translations of $V$ by elements of $V$, and also the group $S \cong \operatorname{Sym}(d)$ consisting of the permutations of $V$ induced by permuting the coordinates of $V$. Since $B$ acts regularly on $V$, and $S$ is the stabiliser of $(0,0, \ldots, 0)$ in $A$, we have $A=S B$ with $S \cap B=1$. In its natural representation, $A$ is a subgroup of $\operatorname{Sym}(V)$ of order $2^{d} d$ ! and degree $2^{d}$. Alternatively, $A$ may be represented as the reflection group consisting of all $d \times d$ monomial matrices with nonzero entries \pm 1 (as a Coxeter group $A$ is denoted by $\left.A_{d-1}\right)$.

The next result follows immediately from (1.1) and (1.2).

(2.1) Let $G$ be a group of order $2^{d}$. Then there exists a generating set $T$ of $G$ with $1 \notin T$ and $T=T^{-1}$ such that Cayley $(G, T)$ is isomorphic to the $d$-cube if and only if $G$ is isomorphic to a regular subgroup $R$ of the group $A$ defined above. The equivalence types $(G, T)$ correspond one-to-one with the $A$-conjugacy classes of regular subgroups of $A$ isomorphic to $G$.

For computational purposes we prefer a more compact representation of $A$. Since $A$ is isomorphic to the wreath product $C_{2}$ wr $\operatorname{Sym}(d)$ it has a faithful permutation representation of degree $2 d$ as well as its natural representation of degree $2^{d}$ (see, for example, [3, Chapter 2]). Specifically, let $\Pi$ be the partition of $\{1,2, \ldots, 2 d\}$ whose parts are the 2-subsets $\{1, d+1\},\{2, d+2\}, \ldots,\{d, 2 d\}$, and let $A_{0}$ be the subgroup of Sym $(2 d)$ consisting of all permutations which leave $\Pi$ invariant. Then $A$ is isomorphic to $A_{0}$ with $B$ corresponding to $B_{0}:=\langle(1, d+1), \ldots,(d, 2 d)\rangle$, and $S$ corresponding to $S_{0}:=\langle(1,2)(d+1, d+2),(1,2, \ldots, d)(d+1, d+2, \ldots, 2 d)) \cong$ Sym $(d)$. It is easily 
verified that $S_{0}$ is the set of all permutations in $\operatorname{Sym}(2 d)$ which leave $\Pi$ invariant and map $\{1,2, \ldots, d\}$ into itself, and that a subgroup $R$ of $A$ is regular (in the natural representation) if and only if its image $R_{0}$ in $A_{0}$ has the properties: (i) $\left|R_{0}\right|=2^{d}$; and (ii) $R_{0} \cap S_{0}=1$. Now (2.1) gives the following.

(2.2) Let $G$ be a group of order $2^{d}$. Then there exists at least one set $T$ of generators of $G$ with $1 \notin T$ and $T=T^{-1}$ such that Cayley $(G, T)$ is isomorphic to the $d$-cube if and only if $G$ is isomorphic to a subgroup $R_{0}$ of $\operatorname{Sym}(2 d)$ such that:

(i) $R_{0}$ leaves the partition $\Pi$ described above invariant; and

(ii) no nontrivial element of $R_{0}$ maps $\{1,2, \ldots, d\}$ into itself.

REMark. In particular, if Cayley $(G, T)$ is isomorphic to the $d$-cube, then $G$ has a faithful permutation representation of degree $2 d$. This shows, for example, that if $k$ is the integer such that $d<2^{k} \leqslant 2 d$ then the orders of the elements of $G$ are bounded by $2^{k}$ and the solvable length of $G$ is bounded by $k$.

\section{ENUMERATING CONJUGACY CLASSES OF REgULAR SUBgROUPS}

We now turn to the question of how to enumerate efficiently the regular subgroups of the automorphism group $A$ of the $d$-cube. By (1.2) we really want to enumerate the $A$-conjugacy classes of such groups. It turns out to be easier to enumerate first the $P$-conjugacy classes of regular subgroups of a Sylow 2-subgroup $P$ of $A$, and then to remove extraneous groups which are $A$-conjugate but not $P$-conjugate. Since the process we describe works equally well for a $p$-group for any prime $p$ we shall describe it in that more general setting.

Problem. Let $p$ be a prime. Given a finite $p$-group $P$ and a subgroup $P_{0}$ of index $p^{d}$, find representatives of the $P$-conjugacy classes of subgroups $R$ of $P$ satisfying:

$$
|R|=p^{d} \text { and } R \cap P_{0}=1 .
$$

REMARK. In our original problem $P$ is a transitive permutation group of degree $2^{d}$, $P_{0}$ is a point stabiliser in $P$, and the subgroups $R$ with property $\left(^{*}\right)$ are the regular subgroups. The more general formulation is helpful because we want to work with the representation of degree $2 d$ described in (2.2).

Since $P$ is a $p$-group, every subgroup is subnormal, so we can find a normal series:

$$
P_{0} \triangleleft P_{1} \triangleleft \ldots \triangleleft P_{d}=P
$$

where $P_{k} / P_{k-1}$ is of order $p$ and generated by $P_{k-1} z_{k}$, say, for $k=1,2, \ldots, d$. Suppose that $R$ is a subgroup of $P$ with properties $\left(^{*}\right)$ and put $R_{k}:=R \cap P_{k}$. Then

$$
1=R_{0} \triangleleft R_{1} \triangleleft \ldots \triangleleft R_{d}=R
$$


is a normal series for $R$ whose factors are each of order dividing $p$. Since $|R|=p^{d}$, each factor in this series has, in fact, order equal to $p$, so we have a composition series for $R$. Thus $\left|R_{k}\right|=p^{k}$ and $P_{k}=P_{0} R_{k}$. In particular, $z_{k} \in P_{0} R_{k}$ and so $u_{k} z_{k} \in R_{k}$ for some $u_{k} \in P_{0}$. Since $u_{k} z_{k} \notin P_{k-1}$, we conclude that $R_{k-1} u_{k} z_{k}$ generates $R_{k} / R_{k-1}$. Note that $u_{k}$ is completely determined by $R$ since $R \cap P_{0}=1$. Hence we can associate with each subgroup $R$ satisfying $\left({ }^{*}\right)$ a unique $d$-tuple $\left(u_{1}, \ldots, u_{d}\right)$ of elements from $P_{0}$ such that

$$
R_{k}=\left\langle u_{1} z_{1}, \ldots, u_{k} z_{k}\right\rangle \text { for } k=0,1, \ldots, d .
$$

Let $\mathcal{U}$ denote the set of all such $d$-tuples as $R$ ranges over the subgroups of $P$ satisfying $\left({ }^{*}\right)$.

(3.1) A $d$-tuple $\left(u_{1}, \ldots, u_{d}\right)$ of elements from $P_{0}$ lies in $\mathcal{U}$ if and only if, for $i=1, \ldots, d$, we have:

(i) $u_{i} z_{i}$ normalises $\left\langle u_{1} z_{1}, \ldots, u_{i-1} z_{i-1}\right\rangle$; and

(ii) $\left(u_{i} z_{i}\right)^{p} \in\left\langle u_{1} z_{1}, \ldots, u_{i-1} z_{i-1}\right\rangle$.

PROOF: It is easy to check that the two conditions are necessary, so assume that (i) and (ii) both hold; we shall show that $\left(u_{1}, \ldots, u_{d}\right) \in \mathcal{U}$.

Put $R_{i}:=\left\langle u_{1} z_{1}, \ldots, u_{i} z_{i}\right\rangle$. Then $1=R_{0} \leqslant R_{1} \leqslant \ldots \leqslant R_{d}$ is a normal series by (i), and the factors have orders dividing $p$ by (ii), so $\left|R_{d}\right| \leqslant p^{d}$. Since $P_{0} R_{d}=$ $\left\langle P_{0}, z_{1}, \ldots, z_{d}\right\rangle=P, R_{d}$ is of order at least $\left|P: P_{0}\right|=p^{d}$. Hence $\left|R_{d}\right|=p^{d}$, and now $P_{0} R_{d}=P$ implies $P_{0} \cap R_{d}=1$. Thus $R_{d}$ satisfies $\left(^{*}\right)$ as required.

Now consider the condition of $P$-conjugacy. Suppose that $\left(u_{1}, \ldots, u_{d}\right)$ and $\left(v_{1}, \ldots, v_{d}\right)$ lie in $\mathcal{U}$ and define subgroups $R$ and $Q$, respectively, which satisfy conditions analogous to $\left({ }^{*}\right)$. Since $P=P_{0} R, R$ is conjugate to $Q$ under $P$ if and only if $R$ is conjugate to $Q$ under $P_{0}$. Suppose $w \in P_{0}$ such that $w^{-1} R w=Q$. Then $w$ normalises $P_{k}$ so $w^{-1} R_{k} w=Q_{k}$ for each $k$. Thus to find the $P$-conjugacy classes of subgroups satisfying $\left({ }^{*}\right)$ in a backtrack program, it is enough to choose exactly one $P_{0}$-conjugacy class of subgroups $R_{k}$ at each stage.

Assume that $k \geqslant 1$. Suppose that $\left(u_{1}, \ldots, u_{k-1}\right)$ is a $(k-1)$-tuple of elements from $P_{0}$ which extends to two $k$-tuples of elements from $P_{0}$, say $\left(u_{1}, \ldots, u_{k}\right)$ and $\left(u_{1}, \ldots, u_{k-1}, v\right)$, both of which satisfy conditions of type (i) and (ii) of (3.1) up to this stage. As before, put $R_{i}:=\left\langle u_{1} z_{1}, \ldots, u_{i} z_{i}\right\rangle$ for $i=0, \ldots, k$, and $Q_{k}:=\left\langle u_{1} z_{1}, \ldots, u_{k-1} z_{k-1}, v z_{k}\right\rangle$.

(3.2) There exists $w \in P_{0}$ such that $w^{-1} R_{k} w=Q_{k}$ if and only if:

(i) $w$ normalises $R_{k-1}$; and

(ii) $R_{k-1} v z_{k}=R_{k-1} w^{-1} u_{k} z_{k}$ w.

PROOF: The conditions are clearly sufficient for $R_{k}$ and $Q_{k}$ to be conjugate under $w$. Conversely, assume $w^{-1} R_{k} w=Q_{k}$ for some $w \in P_{0}$. Then (i) holds because 
$R_{k-1}=R_{k} \cap P_{k-1}=Q_{k} \cap P_{k-1}$. Moreover, $v z_{k} \in Q_{k} \cap P_{k}, u_{k} z_{k} \in R_{k} \cap P_{k}$ and $w \in P_{k-1}$, therefore $w v z_{k} w^{-1} \in R_{k} \cap P_{k-1} u_{k} z_{k}=\left(R_{k} \cap P_{k-1}\right) u_{k} z_{k}=R_{k-1} u_{k} z_{k}$. Condition (ii) now follows because $w$ normalises $R_{k-1}$.

The conditions of (3.1) and (3.2) can be used in a backtrack program to generate a complete set of representatives of $P$-conjugacy classes $\left(=P_{0}\right.$-conjugacy classes) of subgroups of $P$ satisfying $\left({ }^{*}\right)$. The $d$-tuples in $\mathcal{U}$ are generated one element at a time, discarding branches which will lead to $P_{0}$-conjugate subgroups. Suppose that $k \leqslant d$ and that $\left(u_{1}, \ldots, u_{k-1}\right)$ is a $(k-1)$-tuple of elements of $P_{0}$ such that conditions (i) and (ii) of (3.1) hold for $i=1, \ldots, k-1$. Put $R_{k-1}:=\left\langle u_{1} z_{1}, \ldots, u_{k-1} z_{k-1}\right\rangle$. Then in the next step we must construct a subset $W \subseteq P_{0}$ such that

(i) $\left(u_{1}, \ldots, u_{k-1}, u_{k}\right)$ satisfies (i) and (ii) of (3.1) for $i=k$ for each $u_{k} \in W$; and

(ii) $R_{k}:=\left\langle u_{1} z_{1}, \ldots, u_{k} z_{k}\right\rangle\left(u_{k} \in W\right)$ is a complete set of representatives of the $P_{0}$-conjugacy classes of subgroups obtained by extending $R_{k-1}$.

It follows from (3.1) and (3.2) that $W$ has these properties provided the elements $R_{k-1} u z_{k}(u \in W)$ run over a complete set of representatives of the $N_{P_{0}}\left(R_{k-1}\right)$-orbits (under conjugation) of the set of elements of order $p$ of the form $R_{k-1} v z_{k}\left(v \in P_{0}\right)$ in $N_{P_{k}}\left(R_{k-1}\right) / R_{k-1}$.

It may happen that no extension of the $(k-1)$-tuple is possible $(W=\emptyset)$. On the other hand, suppose $W \neq \emptyset$ and choose $u_{k} \in W$.

Put $R_{k}:=\left\langle u_{1} z_{1}, \ldots, u_{k} z_{k}\right\rangle$, and $N_{i}:=N_{P_{0}}\left(R_{i}\right)$ for $i=0, \ldots, k$. Then $N_{k} \leqslant$ $N_{k-1} \leqslant \ldots \leqslant N_{0}=P_{0}$ because $R_{i-1}=R_{i} \cap P_{k-1}$. Indeed, since $u_{k} z_{k}$ normalises $R_{k-1}$, the elements of the form $R_{k-1} v z_{k}\left(v \in P_{0}\right)$ in $N_{P_{k}}\left(R_{k-1}\right) / R_{k-1}$ are precisely those of the form $R_{k-1} u u_{k} z_{k}$ with $u \in N_{k-1}$. Hence we can choose $W$ as any set with the property that $v z_{k}(v \in W)$ is a set of representatives of the $N_{k-1}$-conjugacy classes of elements in $\left\{u u_{k} z_{k} \mid u \in N_{k-1}\right.$ and $\left.\left(u u_{k} z_{k}\right)^{p} \in R_{k-1}\right\}$. Note that $R_{k-1} N_{k-1} u_{k} z_{k}$ is invariant under $N_{k-1}$. Indeed, if $w \in N_{k-1}$, then $w^{-1} u_{k} z_{k} w$ lies in $P_{k-1} u_{k} z_{k}$ (since $\left.P_{k-1} \triangleleft P_{k}\right)$ as well as in $N_{P}\left(R_{k-1}\right)$. Thus $w^{-1} u_{k} z_{k} w \in R_{k-1} N_{k-1} u_{k} z_{k}$ as required because $N_{P}\left(R_{k-1}\right) \cap P_{k-1}=R_{k-1} N_{k-1}$.

EXAMPLE 2. We explain how to enumerate the Cayley graphs isomorphic to the 3cube. In this case we can take $P:=\langle(1,2,4,5),(1,4),(3,6)\rangle$ of order 16 as a Sylow 2-subgroup of $\operatorname{Sym}(6)$ which leaves the partition $\Pi:=\{\{1,4\},\{2,5\},\{3,6\}\}$ invariant. The subgroup $P_{0}:=\langle w\rangle \leqslant P$ with $w:=(1,2)(4,5)$ has index 8 , and corresponds to a point stabiliser in the representation of degree $2^{3}$ of $P$ (see (2.2)). If we take $T:=\{(1,4),(2,5),(3,6)\}$, then $Q:=\langle T\rangle$ is an elementary Abelian normal subgroup of order 8 in $P$ with $Q \cap P_{0}=1$. Indeed Cayley $(Q, T)$ gives the standard representation of the 3-cube. We next find suitable elements, $z_{1}:=(3,6), z_{2}:=(1,4)(2,5)$ and 
$z_{3}:=(2,5)$, so that $1 \triangleleft\left\langle z_{1}\right\rangle \triangleleft\left\langle z_{1}, z_{2}\right\rangle \triangleleft\left\langle z_{1}, z_{2}, z_{3}\right\rangle=T$ is a $P_{0}$-composition series for $T$. We must now find the 3-tuples $\left(u_{1}, u_{2}, u_{3}\right)$ with $u_{i} \in P_{0}$ for which $\left\langle u_{1} z_{1}, u_{2} z_{2}, u_{3} z_{3}\right\rangle$ has order 8. Since $z_{1}, z_{2}, z_{3}$ and $w$ all commute except for the relation $z_{3}^{-1} w z_{3}=w z_{2}$, we find using (3.1) that the only 3 -tuples with the required property are $(I, I, I)$, $(I, I, w),(w, I, I)$ and $(w, I, w)$. It can be checked using (3.2) that the corresponding groups, say $G_{i}(i=1, \ldots, 4)$, are not $P_{0}$-conjugate. In order to choose a set $T_{i}$ of generators for $G_{i}$ so that Cayley $\left(G_{i}, T_{i}\right)$ is isomorphic to the 3-cube $(i=1, \ldots, 4)$ we refer back to $T$. We must choose $T_{i} \subseteq G_{i}$ to consist of three elements such that right multiplication by the $j$ th element of $T_{i}$ maps $P_{0}$ onto $P_{0} t_{j}$ where $t_{j}$ is the $j$ th element in $T$. This gives $T_{1}=\left\{z_{2} z_{3}, z_{3}, z_{1}\right\}, T_{2}=\left\{w z_{3} z_{2}, w z_{3}, z_{1}\right\}, T_{3}=\left\{z_{2} z_{3}, z_{3}, w z_{1}\right\}$ and $T_{4}=\left\{w z_{3} z_{2}, w z_{3}, w z_{1}\right\}$ and so we obtain the four Cayley graphs listed in Example 1 . Since the pairs $\left(G_{i}, T_{i}\right)$ are obviously mutually inequivalent, we are finished.

\section{CAYley gRAPHS ISOMORPhiC to THE $d$-CUBE}

Example 2 shows how to enumerate the Cayley graphs isomorphic to the 3-cube. For $d>3$ the procedure is similar. It can be described as follows.

We start with a Sylow 2-subgroup $P$ of Sym(2d) leaving the partition $\Pi:=$ $\{\{1, d+1\},\{2,2+d\}, \ldots,\{d, 2 d\}\}$ invariant, and the subgroup $P_{0}$ of $P$ consisting of the permutations which map $\{1, \ldots, d\}$ into itself. Then $Q:=\langle(1, d+1),(2,2+d), \ldots$ $(d, 2 d)\rangle$ is an elementary Abelian normal subgroup of $P$ of order $2^{d}$ such that $P=$ $P_{0} Q$ and $Q \cap P_{0}=1$. We can therefore choose $z_{i}(i=1, \ldots, d)$ in $Q$ such that $1 \triangleleft\left\langle z_{1}\right\rangle \triangleleft \ldots \triangleleft\left\langle z_{1}, z_{2}, \ldots, z_{d}\right\rangle=Q$ is a $P_{0}$-composition series for $Q$. With these values of $z_{i}$ we carry out the backtrack program sketched in Section 3 to find a complete set of representatives for the $P$-conjugacy classes of subgroups $R$ of $P$ which satisfy the condition $\left(^{*}\right)$. For each of these subgroups we find a set $T_{R}$ of generators such that Cayley $\left(R, T_{R}\right)$ is isomorphic to the $d$-cube. Finally extraneous pairs $\left(R, T_{R}\right)$ which are equivalent to other pairs in our list are eliminated, first using a crude criterion based on conjugacy classes to distinguish groups which are obviously not isomorphic, and then using (1.3) to decide equivalence in the remaining undetermined cases.

All calculations were done using GAP 3.1 [11].

\subsection{The Cayley graphs isomorphic to the 4-Cube.}

For $d=4$, we obtained $22 P$-conjugacy classes of subgroups $R$ satisfying $\left(^{*}\right)$. These 22 classes yielded 14 equivalence classes of Cayley graphs isomorphic to the 4cube. Generators for representatives of these classes are given by:

$$
\begin{aligned}
& {[a 4, c 4, d 4, e 4],[b 4, f 4, g 4, h 4],[c 4, d 4, e 4, f 4],[a 4, b 4, g 4, h 4],[i 4, j 4, k 4, l 4],} \\
& {[e 4, f 4, i 4, k 4],[a 4, h 4, m 4, n 4],[c 4, d 4, j 4, l 4],[b 4, g 4, o 4, p 4],[e 4, f 4, m 4, n 4],} \\
& {[a 4, h 4, i 4, k 4],[c 4, d 4, o 4, p 4],[q 4, r 4, s 4, t 4],[e 4, n 4, u 4, v 4]}
\end{aligned}
$$


where

$$
\begin{aligned}
a 4 & :=(3,7), b 4:=(4,8), c 4:=(2,4,6,8), d 4:=c 4^{-1}, e 4:=(1,5)(2,4)(6,8), \\
f 4 & :=(2,4)(3,7)(6,8), g 4:=(2,6), h 4:=(1,5), i 4:=(1,3)(2,4,6,8)(5,7), \\
j 4 & :=(1,3,5,7)(2,4)(6,8), k 4:=i 4^{-1}, l 4:=j 4^{-1}, m 4:=(1,3)(4,8)(5,7), \\
n 4 & :=(1,3)(2,6)(5,7), o 4:=(1,3,5,7), p 4:=o 4^{-1}, q 4:=(1,2,3,4,5,6,7,8), \\
r 4 & :=(1,2,7,8,5,6,3,4), s 4:=r 4^{-1}, t 4:=q 4^{-1}, u 4:=(1,2,3,8,5,6,7,4), v 4:=u 4^{-1} .
\end{aligned}
$$

Many of the groups involved are isomorphic. For example, the first group is isomorphic to the second via the mapping $a 4 \mapsto h 4, c 4 \mapsto a 4 c 4$ and $e 4 \mapsto b 4$.

\subsection{The Cayley graphs isomorphic to The 5-Cube.}

For $d=5$, we obtained $68 P$-conjugacy classes of subgroups $R$ satisfying (*). These 68 classes yielded 45 equivalence classes of Cayley graphs isomorphic to the 5cube. Generators for representatives of these classes are given by:

$[a 5, c 5, b 5, d 5, e 5],[f 5, g 5, h 5, d 5, i 5],[d 5, j 5, l 5, k 5, m 5],[n 5, o 5, p 5, d 5, q 5]$, $[c 5, d 5, j 5, k 5, e 5],[g 5, r 5, d 5, s 5, i 5],[a 5, b 5, d 5, l 5, m 5],[f 5, h 5, t 5, d 5, u 5]$, $[c 5, r 5, d 5, s 5, e 5],[g 5, d 5, j 5, k 5, i 5],[a 5, b 5, t 5, d 5, u 5],[v 5, x 5, d 5, y 5, w 5]$, $[c 5, z 5, r 5, s 5, e 5],[z 5, a a 5, b b 5, c c 5, d d 5],[a 5, b 5, z 5, t 5, u 5],[z 5, v 5, x 5, y 5, w 5]$, $[z 5, a a 5, s 5, b b 5, e 5],[e e 5, a 5, c 5, b 5, e 5],[f 5, g 5, e e 5, h 5, i 5],[e e 5, j 5, l 5, k 5, m 5]$, $[e e 5, r 5, t 5, s 5, u 5],[f 5, e e 5, c 5, h 5, e 5],[g 5, e e 5, a 5, b 5, i 5],[e e 5, r 5, l 5, s 5, m 5]$, $[e e 5, t 5, j 5, k 5, u 5],[e e 5, c 5, j 5, k 5, e 5],[g 5, e e 5, r 5, s 5, i 5],[e e 5, a 5, b 5, l 5, m 5]$, [f $5, e e 5, h 5, t 5, u 5],[e e 5, c 5, r 5, s 5, e 5],[g 5, e e 5, j 5, k 5, i 5],[f 5, e e 5, h 5, l 5, m 5]$, $[e e 5, a 5, b 5, t 5, u 5],[g 5, e e 5, a 5, b 5, e 5],[f 5, e e 5, c 5, h 5, i 5],[f f 5, f 5, g 5, h 5, i 5]$, $[f f 5, j 5, l 5, k 5, m 5],[f f 5, c 5, j 5, k 5, e 5],[f f 5, a 5, b 5, l 5, m 5],[f f 5, f 5, h 5, t 5, u 5]$, $[f f 5, a a 5, s 5, b b 5, e 5]$

where

$$
\begin{aligned}
a 5 & :=(2,4,7,9), b 5:=a 5^{-1}, c 5:=(2,4)(3,8)(7,9), d 5:=(1,3)(2,4)(5,10)(6,8)(7,9), \\
e 5 & :=(1,6)(2,4)(7,9), f 5:=(4,9), g 5:=(3,8), h 5:=(2,7), i 5:=(1,6), \\
j 5 & :=(1,3)(2,4,7,9)(6,8), k 5:=j 5^{-1}, l 5:=(1,3,6,8)(2,4)(7,9), m 5:=l 5^{-1}, \\
n 5 & :=(1,2)(3,4,8,9)(6,7), o 5:=n 5^{-1} p 5:=(1,2,6,7)(3,4)(8,9), q 5:=p 5^{-1}, \\
r 5 & :=(1,3)(4,9)(6,8), s 5:=(1,3)(2,7)(6,8), t 5:=(1,3,6,8), u 5:=t 5^{-1}, \\
v 5 & :=(1,2,3,4,6,7,8,8), w 5:=v 5^{-1}, x 5:=(1,2,8,9,6,7,3,4), y 5:=x 5^{-1}, \\
z 5 & :=(1,2)(3,4)(5,10)(6,7)(8,9), a a 5:=(1,2,3,9,6,7,8,4), b b 5:=a a 5^{-1}, \\
c c 5 & :=(1,4,3,2,6,9,8,7), d d 5:=c c 5^{-1}, e e 5:=(2.4)(5,10)(7,9), f f 5:=(5,10) .
\end{aligned}
$$




\title{
4.3 The Cayley graphs isomorphic to the 6-Cube.
}

For $d=6,894 P$-conjugacy classes of subgroups $R$ satisfying $\left({ }^{*}\right)$ were obtained. These 894 classes yielded 238 equivalence classes of Cayley graphs isomorphic to the 6-cube.

\section{REFERENCES}

[1] L. Babai, 'Isomorphism problem for a class of point-symmetric structures', Acta Math. Acad. Sci. Hungar. 29 (1977), 329-336.

[2] N. Biggs, Algebraic graph theory (Cambridge University Press, Cambridge, 1974).

[3] J.D. Dixon and B. Mortimer, Permutation groups (Springer-Verlag, Berlin, Heidelberg, New York, 1996).

[4] E. Dobson, 'Isomorphism problem for Cayley graphs of $\mathbb{Z}_{p}^{3}$, Discrete Math. 147 (1995), 87-94.

[5] X.G. Fang and M.Y. Xu, 'On isomorphisms of Cayley graphs of small valency', Algebra Colloq. 1 (1994), 67-76.

[6] A. Joseph, 'The isomorphism problem for Cayley digraphs on groups of prime-squared order', Discrete Math 141 (1995), 173-183.

[7] P. Kulasinghe and S. Bettayeb, 'On the multiply-twisted hypercube', in Parallel and Distributed Computing (Montreal, $P Q, 1994$ ), Lecture Notes in Computer Science 805 (Springer-Verlag, Berlin, Heidelberg, New York, 1994), pp. 267-278.

[8] A. Lubotzky, 'Cayley graphs: eigenvalues, expanders and random walks', in Surveys in Combinatorics, 1995 (Stirling), London Math. Soc. Lecture Notes 218 (Cambridge University Press, Cambridge, 1995), pp. 155-189.

[9] B.D. McKay and C.E. Praeger, 'Vertex transitive graphs which are not Cayley graphs I', J. Austral. Math. Soc. Ser. A. 56 (1994), 53-63.

[10] S.T. Schibell and R.M. Stafford, 'Processor interconnection networks from Cayley graphs', Discrete Appl. Math. 40 (1992), 333-357.

[11] M. Schönert et al, GAP - Groups, Algorithms and Programing, Lehrstuhl D. für Math. (Rheinisch Westfälische Technische Hochschule, Germany, 1994).

[12] G. Zmor, 'Hash functions and Cayley graphs', Des. Codes Cryptogr. 4 (1994), 381-394.

\author{
Department of Mathematics and Statistics \\ Carleton University \\ Ottawa \\ Canada K1S 5B6
}

